Casos Clínicos

Arch. Esp. Urol. 2010; 63 (6): 460-464

\section{FÍSTULA ENTEROCUTÁNEA COMO COMPLICACIÓN DE NEFROLITOTOMIAA PERCUTÁNEA EN PACIENTES CON CIRUGIAA BARIÁTRICA PREVIA - PRESENTACIÓN DEL CASO Y REVISIÓN DE LA LITERATURA}

\author{
Gustavo Marquesine Paul, Luiz Edison Slongo y Luiz \\ Carlos de Almeida Rocha.
}

Hospital de Clínicas. Universidade Federal do Paraná. Curitiba. Paraná. Brasil.

Resumen.- OBJETIVO: La fístula enterocutánea es una complicación rara en nefrolitotomía percutánea (NLPC). Son factores de riesgo que contribuyen en su aparición el riñón en herradura, el colon retrorrenal y la dilatación colónica crónica de origen diverso; entre ésta destacamos el postoperatorio tardío de "by-pass" yeyunoileal como tratamiento de la obesidad mórbida.

MÉTODO: Mujer de 35 años con cálculo de 2,5 cm en pelvis renal derecha en el postoperatorio tardío de cirugía bariátrica, técnica de "switch" duodenal, con pérdida hasta el momento de $55 \mathrm{~kg}$ de peso. Sometida a NLPC con dos punciones, la primera, en cáliz inferior que se perdió durante la dilatación y la segunda en el cáliz superior

\section{CORRESPONDENCIA}

Gustavo Marquesine Paul Hospital de Clínicas

Universidade Federal do Paraná Curitiba. Paraná. (Brasil)

i.urologia@terra.com.br

Aceptado para publicar: 16 de febrero 2009 con éxito. Durante el postoperatorio inmediato se observó secreción entérica por la primera punción. Descartada lesión intraperitoneal se optó por tratamiento conservador con drenaje de la fístula, antibioticoterapia de amplio espectro, ayuno y nutrición parenteral total. El débito de la fístula cesó al octavo día del postoperatorio retirándole el drenaje y el tubo de nefrostomía; en el décimo día fue alta hospitalaria. El control ambulatorio de la paciente muestra que se halla en buen estado.

RESULTADOS: Se observó un caso de fístula enterocutánea por NLPC durante el postoperatorio tardío by-pass yeyunoileal, tratado con éxito con terapia conservadora. Varios autores apuntan a la distensión colónica crónica y a la gran pérdida de grasa peritoneal como causas del aumento de punciones colónicas inadvertidas en el acceso renal percutáneo.

CONCLUSIONES: El riesgo de que se desarrollen complicaciones con fístula enterocutánea es significativamente mayor en un paciente con cirugía bariátrica previa que en los demás.

Palabras clave: Nefrolitíasis. Fístula intestinal. Perforación intestinal.

Summary.- OBJECTIVE: Enterocutaneous fistula is a rare complication after percutaneous nephrolithotomy. Some situations are risk factors for it to occur, such as horseshoe kidney, retrorenal colon and chronic colonic dilation by several factors, among them late postoperative time after yeyunoileal bypass in morbid obesity treatment.

METHODS: 35-year-old female patient with a $2.5 \mathrm{~cm}$ calculus in the right renal pelvis. Late postoperative time after bariatric surgery using duodenal switch technique, and $55 \mathrm{~kg}$ lost till present. She undergone percutaneous nephrolithotomy (PCNL) with two punctures, one in the lower calyx, lost during dilation, and other in the upper calyx through which surgery was successfully carried out. During immediate postoperative time it enteric secretions were observed through the lower chalice puncture. Once the intraperitoneal lesion was removed, decision for conservative treatment was taken with fistula drainage, broad-spectrum antibiotic therapy, fasting and total parenteral nutrition. The fistula debt ceased in the 8th postoperative (PO) day, when drain and nephrostomy tube were withdrawn and patient was discharged from the hospital on 10th PO day. On ambulatory follow up the patient is in good conditions.

RESULTS: One case of enterocutaneous fistula after PNL presented in a patient in late postoperative time after yeyunoileal bypass. She was successfully treated by conservative therapy. Several authors point to chronic colonic distention and to great loss of retroperitoneal fat as causes for the increase of inadvertent colonic punctures in percutaneous renal access. 
CONCLUSIONS: The risk of complications with enterocutaneous fistula is significantly higher during the post operatory in patients of bariatric surgery than in others.

Keywords: Nephrolithiasis. Intestinal fistula. Intestinal perforation.

\section{INTRODUCCIÓN}

La lesión iatrogénica del colon es una complicación infrecuentemente comunicada en cirugía renal percutánea (NLPC); en la literatura inglesa, no más de 25 casos (1). El diagnóstico de este tipo de iatrogenia se caracteriza por una gran variabilidad de señales y síntomas que pueden ocurrir tanto en la intervención quirúrgica como en el postoperatorio inmediato o precoz. La lesión colónica no reconocida puede llevar a la formación de absceso retroperitoneal o fístula (nefrocolónica o colocutánea) (1). La presencia de heces en el espacio intraperitoneal puede desarrollar una peritonitis (2). Otra potencial presentación de esta lesión es la septicemia. A pesar de la exploración y reparación del daño colónico llevados a cabo en algunos casos, el tratamiento no qui-

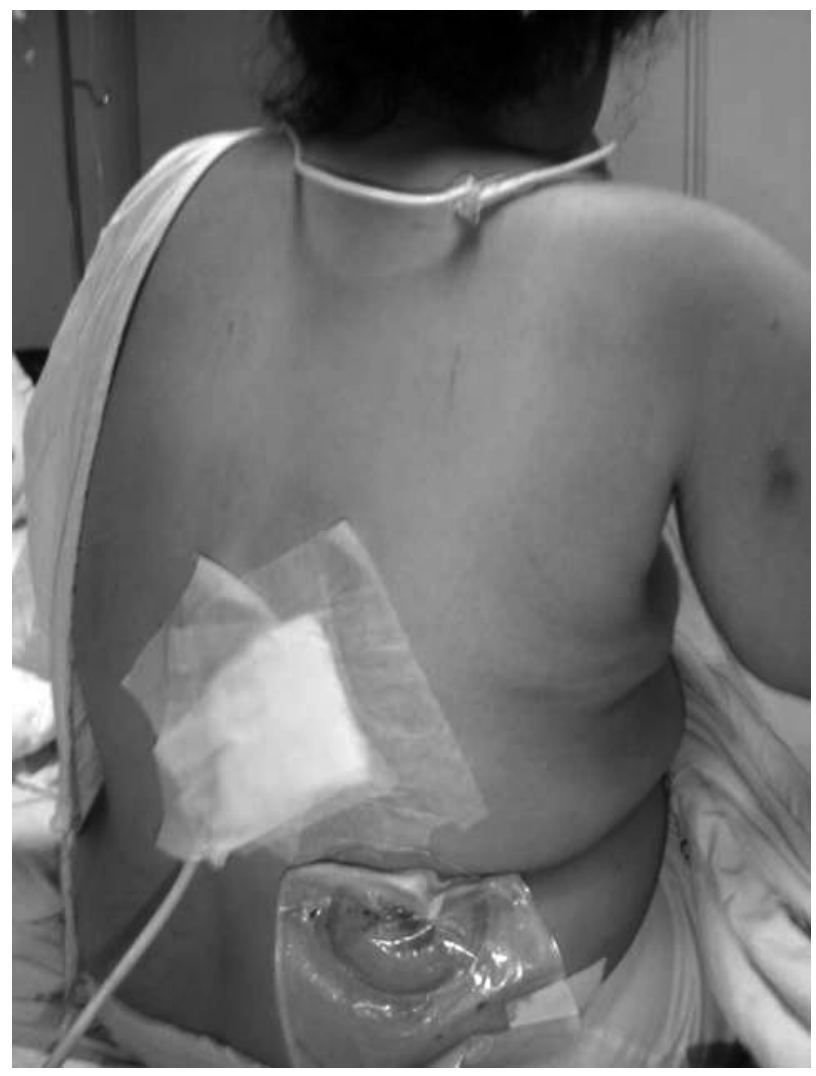

FIGURA 1. Posicionamiento de nefrostomía y de fístula reno-cutanea. rúrgico es preferible cuando no hay paso del material entérico en el peritoneo. Algunas condiciones pueden incrementar el riesgo de que ocurran lesiones colónicas en cirugía percutánea, como condiciones crónicas de distensión del colon (tras by pass yeyunoileal en cirugía de corrección de obesidad), cirugía renal previa y mielomeningocele (1).

\section{CASO CLÍNICO}

Paciente E. C., mujer, 30 años, con queja de dolor lumbar derecha con dos años de evolución. En la exploración diagnóstica con urografía excretora se identificaron cálculos renales de $2 \times 3 \mathrm{~cm}$ en pelvis renal derecha y fragmentos litiásicos, en cálices renales inferior y medio, resultantes de la fragmentación previa del cálculo en litotripsia extracorpórea (LEOC). Paciente con histórico de obesidad mórbida con realización de cirugía bariátrica (Duodenal Switch), hace tres años, con pérdida de $55 \mathrm{~kg}$ durante el período e índice de masa corporal (IMC) actual de 31. Indicada NLPC que se realizó en decúbito dorsal. La primera punción se hizo en cáliz inferior del riñón derecho con punción cutánea medial a la línea axilar posterior (Figura 1). Trayecto dilatado con dilatador telescopado de Alken y ulterior introducción de vaina de Amplatz y nefroscopio, no se identificó el sistema colector, se observó solamente grasa perirenal. Se abandonó este acceso y se hizo una nueva punción en cáliz superior del mismo riñón, por donde fueron extraídos todos los cálculos. Posicionada nefrostomía en la punción del cáliz superior, por donde, al final del procedimiento, se realizó una pielografia que reveló nefrostomía bien posicionada y ausencia de otras alteraciones significativas (Figura 2). En la punción del cáliz inferior se dejó drenaje de Penrose numero dos para evitar la formación de colección local por el liquido de irrigación utilizado.

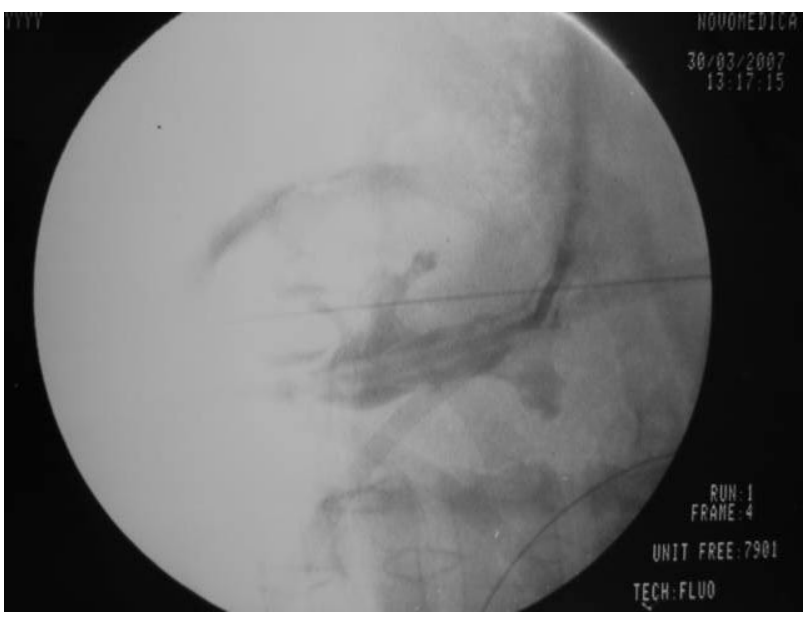

FIGURA 2. Pielografía trans-operatoria. 
A las 11 horas del postoperatorio se observó el extravasado de secreción de aspecto entérico (confirmado tras análisis en laboratorio de lipasa y amilasa) por el drenaje de Penrose. También se observó prostración, fiebre y una caída en el estado general de la paciente que indicaba la presencia de una fístula colocutánea. Se realizaron radiografías de rutina de abdomen agudo y tomografía axial computadorizada (TAC) de abdomen y pelvis. No se observó la presencia de gas, líquido libre o contraste en el espacio intraperitoneal, pero sí de cantidades pequeñas de gas y contraste en espacio perirenal (Figura 3). Se realizó nueva pielografia anterógrada por la nefrostomía que no observó comunicación del sistema colector con el trato digestivo (Figura 4).

Se inició tratamiento conservador con ayuno, antibioticoterapia de amplio espectro (ceftriaxona y metronidazol endovenoso, ambos $2 \mathrm{~g} /$ día), nutrición parenteral total (NPT) por catéter venoso central y observación clínica cuidadosa con cuantificaciones diarias de electrólitos, vitaminas, proteínas y lípidos (lipidograma). NPT con $1000 \mathrm{kcal} /$ día durante los dos primeros días y $2000 \mathrm{kcal} /$ día a partir del tercer día.

Durante la evolución, la paciente presentó mejoría clínica y analítica, con desaparición de fiebre, mejora del hemograma y del estado general, disminución gradual del débito fistuloso que cesó al noveno día del postoperatorio. Se realizó entonces TAC de control que no evidenció colección retroperitoneal alguna. Se retiró el acceso venoso central, el drenaje de Penrose de la fístula y la nefrostomía, también se retornó gradualmente a la dieta vía oral. Al decimosegundo día de postoperatorio le fue dada el alta hospitalaria, con antibioticoterapia vía oral de amplio espectro por más diez días.

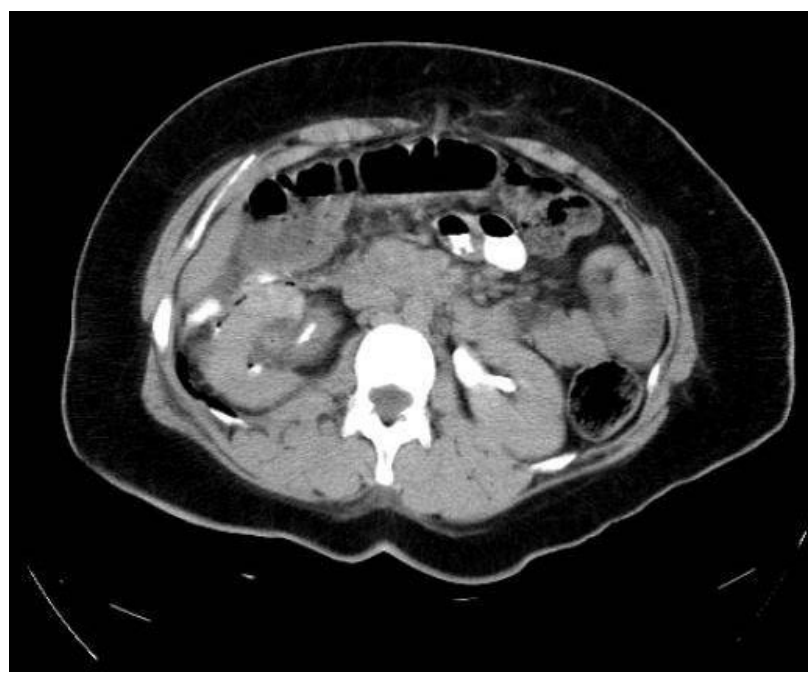

FIGURA 3. Tomografía postoperatoria mostrando contraste retroperitoneal

\section{DISCUSIÓN}

La nefrolitotomía percutánea es una técnica quirúrgica segura y factible para el tratamiento de cálculos renales. Esta técnica reemplazó la cirugía abierta como el tratamiento elegido para cálculos renales grandes (con más de $2 \mathrm{~cm}$ ) o múltiplos. Posee una tasa de 3 - $4 \%$ de complicaciones postoperatorias (3) tales como: fístula arteriovenosa, hemorragia, choque séptico, laceración del parénquima renal, de la pelvis y del uréter, además de lesión colónica con la formación de abscesos retroperitoneales o fístulas colocutáneas. Esta ultima complicación con una incidencia del orden de 0,2 - 0,5\%.

Su cuadro clínico es variable y oscila desde síntomas suaves con fiebre baja, íleo paralítico o leucocitosis hasta síntomas más graves como fecaluria, neumaturia, hemorragia retal y peritonitis.

La incidencia de lesión colónica es más elevada en el lado izquierdo, en punciones hechas en el cáliz renal inferior y en punciones laterales a la línea axilar posterior. La aparición de este tipo de lesiones prevalece en pacientes del sexo femenino, delgados, con riñón en herradura, con distensión colónica crónica por by pass yeyunoileal previo o con cirúgia renal prévia (1). Entre

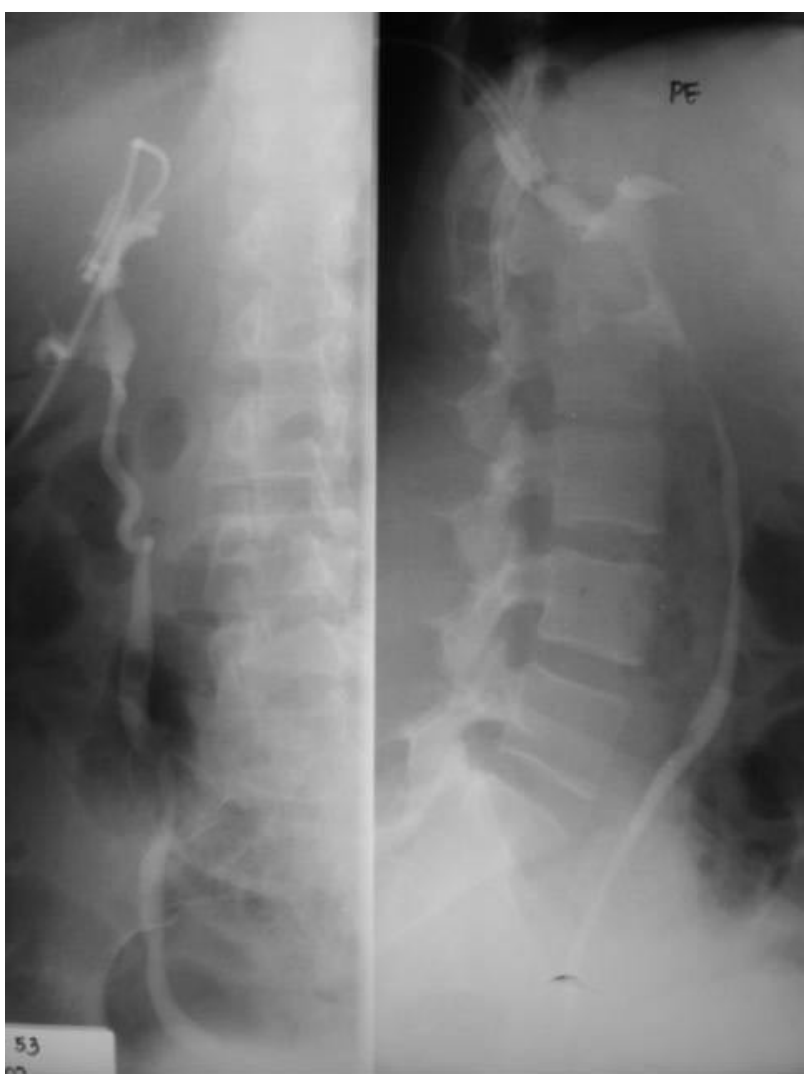

FIGURA 4. Pielografía post-operatoria 
5039 NLPC se observaron 15 casos de perforación colónica, entre ellos, solamente cinco de colon derecho y en cuatro de estos casos se trataba de pacientes con factor de riesgo para lesiones colónicas como riñón en herradura o distensión colónica crónica por by pass intestinal previo (uno y tres casos respectivamente) (1).

La elevación lateral, del paciente, de $30^{\circ}$ del lado de la punción se emplea por algunos autores para desplazar medialmente el colon ipsilateral, disminuyendo, de esta forma, el riesgo de lesión durante la punción (4).

Lesiones colónicas intraperitoneales tras NLPC requieren exploración quirúrgica inmediata y reparación de la lesión. Afortunadamente estos casos son raros. Entre 18 casos de lesión colónica en NLPC, tres necesitaron tratamiento quirúrgico, dos por peritonitis y uno por hemorragia severa y solamente en uno se diagnosticó radiograficamente lesión colónica intraperitoneal (4).

La etiología mas importante de la lesión colónica percutánea es la posición retrorrenal o posterolateral del colon en relación al riñón. La posisión retrorrenal del colon se observa en $4,7 \%$ de las TAC abdominales en posición de pronación (5). Otra observación es la situación más posterior del colon descendente en el polo renal inferior izquierdo. Estos datos pueden explicar la mayor incidencia de lesión colónica en el lado izquierdo y en punciones de cáliz inferior.

La dilatación colónica crónica por cirugía bariátrica previa es un importante factor de riesgo para la aparición de lesión colónica inadvertida durante NLPC (6). En pacientes con este factor de riesgo la gran pérdida de peso concomitante a la reducción de la grasa perirenal puede provocar la translocación del colon en posición retrorrenal antes ocupada por grasa.

La identificación de un colon retrorrenal es de gran importancia para la prevención de la perforación colónica en NLPC. TAC preoperatorio en posición prono determinará la relación anatómica entre el colon y el riñón (7). La baja incidencia de colon retrorrenal, el alto coste del examen y la exposición indiscriminada del paciente a la radiación contraindican la realización de rutina de TCA en todos pacientes antes de NLPC. Pero la TCA puede ser útil en el preoperatorio de NLPC en pacientes con alto riesgo de colon retrorrenal como cirugía renal previa, riñón en herradura, distensión colónicas crónicas, mielomeningocele y postoperatorio de by pass yeyuno ileal (7).

Son muchos los métodos descritos para evitar la perforación del colon durante NLPC. Alken y colaboradores recomiendan la realización de la punción renal dirigida por ultrasonografía. Matlague y colaboradores (2003) realizan la punción guiada por tomografía. Tras la identificación de la situación colónica entre la piel y el riñón se puede determinar una nueva ubicación para la punción cutánea. En esta situación, el acceso renal por el polo superior también se torna una alternativa (6).

El diagnóstico y el manejo precoces de la lesión son la clave para evitar complicaciones de la perforación colónica. La mayoría de los autores recomiendan que se haga una pielografía anterógrada al cabo de cada NLPC para rastrear una posible perforación colónica no percibida durante el procedimiento. Alternativamente, el diagnóstico postoperatorio puede ser hecho con TAC de abdomen o por pielografía retrógrada (1). Tras el diagnóstico, el primer paso es deshacer la conexión entre el aparato urinario y el sistema digestivo. Normalmente se e utiliza el acceso de un catéter doble J por vía retrógrada y se reemplaza la nefrostomía por un drenaje de Penrose para facilitar el drenaje externo de la fístula (1). Otros autores citan la extracción de la nefrostomía de la vía excretora y su posicionamiento retroperitoneal pericolónico para que sirva de drenaje quirúrgico para la fístula (9).

El tratamiento exitoso de pacientes con fístula entero cutánea requiere: tratamiento antiséptico, prevención del desequilibrio hidroelectrolítico, protección de la piel al rededor de la fístula, la nutrición adecuada del paciente y que se controle el drenaje de la fístula.

El control del débito fistuloso se realiza mas fácilmente por el sondaje de su trayecto con un drenaje. La NPT fue un avance importante en el tratamiento de pacientes con fístulas intestinales pues juega un papel fundamental en la prevención de la desnutrición. El ocreotide, análogo a la somatostatina de larga acción, se utiliza con éxito para la disminución del débito fistuloso en pacientes con fístula enterocutánea, especialmente en pacientes con fístula de alto débito (10). La mayoría de las fístulas cicatrizará espontáneamente tras cuatro, cinco o seis semanas de tratamiento conservador. En el caso en que la fístula no se cierre en este periodo, una intervención quirúrgica puede ser indicada.

\section{CONCLUSIÓN}

La perforación colónica durante la NLPC es una complicación rara que puede evolucionar con el desarrollo de fístula colocutánea. Esta lesión es más frecuente en punciones orientadas hacia el riñón izquierdo y hacia los cálices inferiores. Se observó que condiciones como cirugía renal previa, riñón en herradura, mielomeningocele $y$, más recientemente, la cirugía bariátrica con by pass yeyuno ileal incrementan el riesgo de la aparición de este tipo de lesiones. Su tratamiento generalmente es conservador, con ayuno, control hidroelectrolítico, antibioticoterápia de amplio espectro y nutrición parenteral total. Se indica el tratamiento quirúrgico en situaciones particulares como cuando hay extravasamiento colónico intraperitoneal o cuando la fístula no se cierra con el tratamiento conservador. 


\section{BIBLIOGRAFÍA Y LECTURAS RECOMENDADAS (*lectura de interés $y^{* *}$ lectura fundamental)}

**1. El-Nahas AR, Shokeir AA, El-Assmy AM, Shoma AM, Eraky I, El-Kenawy MR, et al. Colonic perforation during perctaneous nephrolithotomy: study of risk factors. Urology 2006; 67:937-41.

2. Wolf Jr, J.S. Management of intraoperative diagnosed colonic injury during perctaneous nephrolithotomy. Tech Urol 1988; 4:160-4.

3. El-Kanawy MR, El-Kappany HA, El-Diasty TA, Ghoneim MA. Perctaneous nephrolithotomy for renal stones in over 1000 patients. Br J Urol 1992; 69:470-5.

*4. Gerspach JM, Bellman GC, Stoller ML, Fugelso P. Conservative management of colon injury following percutaneous renal surgery. Urology 1997; 49:8316.

5. Hopper KD, Sherman JL, Gaudier FA. The retrorenal colon in supine and prone position. Radiology 1987; 162:443-6.

**6. Clayman R. Urolithiasis, endourology and laparoscopy. J Urol 2007; 177:1366. es solamente una pagina

7. Skoog SJ, Reed MD, Gaudier FA, Dunn, NP. The posterior and retrorenal colon: implication in percutaneous nephrolithotomy. J Urol 1985; 134:110-2.

*8. Matlage BR, Shah OD, Zagoria RJ. Computadorized tomography guided acess for perctaneous nephrolithotomy. J Urol 2003; 170:45-7.

9. Lê Roy AJ, Williams HJ, Bender CE, Segura JW; Patterson DE; Benson RC. Colon perforation following perctaneous nephrolithotomy and renal calculus removal. Radiology 1985; 155:83-5.

10. Sancho JL, di Constanzo J, Nubiola P, Larrad A, Beguiristain A, Roqueta F. et al. Randomized doubleblind placebo-controlled trial of early octreotide patients in postoperative enterocutaneous fistula. $\mathrm{Br}$ J Surg 1995; 82:638-41.

11. Fernández González I, Serrano Pascual A, García Cuerpo E, Berenguer Sánchez A, Lovaco Castellano F. Técnica endourológica anterógrada y retrógrada. Instrumental y metodología. Arch Esp Urol 2004; 57:251-8.

12. Gallo Rolanía FJ; Beneitez Alvarez ME. El papel actual de la cirugía percutánea renal en un Servicio de Urología General. Arch Esp Urol 2001; 54:1111-6. 